\title{
A Cognitive Approach to the Semantics in the Sacred Context: Semantic and Symbolic Function of Sacred Words
}

\author{
Rajdeep Singh ${ }^{1}$ \\ ${ }^{1}$ Department of Linguistics, Payame Noor University, Tehran, Iran \\ Correspondence: Rajdeep Singh Department of Linguistics, Payame Noor University, Tehran, Iran.
}

Received: June 11, 2018

Accepted: July 22, 2018

Online Published: August 6, 2018

doi:10.5430/elr.v7n3p1

URL: http://dx.doi.org/10.5430/elr.v7n3p1

\begin{abstract}
One of the intriguing features of language interaction with society and culture is the position of certain words as sacred within that society. Thus, it is important to analyze the social process through which sacred words present their particular features. In this paper, we show how sacred words gain their symbolic prominence. Furthermore, we propose a cognitive-semantic model based on the hypothesis of historic automaticity chain that explains well the reason behind the loss of semantics of the sacred words. In this paper, we compare some sacred words across many Indo-European languages and analyze how the very same sacred words lost ground to other words and became almost empty of semantics and word origin, while still preserving the symbolic notion. This study brings the notion of abstraction to the sacred word framework and clarifies the ways the mind processes sacred semantics. In order to support our hypothesis, we performed two small-scale psycho-linguistic experiments and the results confirmed our hypothesis.
\end{abstract}

Keywords: semantics, cognitive process, symbolic, function, sacred words, experiment, sociolinguistics

\section{Semantic and Symbolic Function of Sacred Words}

Sacredness of words has always been a vehicle to transfer values and moralities from one culture to another, from one faith to another, and so on. However, there is a clear evidence of the difficulty to translate sacred words. It is therefore important to investigate the reasons behind this apparent loss of words while translating sacred words from one language to another. It is even more interesting when the basic sacred words are hard to explain for their followers and the semantics seems missing in the whole definition. Most often sacred words have obscure origin and linguists after years of research still have hard times to find the very origin of such words used in a daily fashion by millions. When we look at the sacred context, rituals and religious ceremonies present us with a realm of sacred words which semantics seem hard to explain and to define. Tambiah (1968) investigates the importance and position of words in ritual contemporary anthropology and analyzes the intelligibility of sacred words to cultures wherein these words were first formed. Veal and Hao (2008) investigate metaphor as a phenomenon that manipulates the conventions of semantic description, and present a computational model that is as flexible as the metaphor in order to interpret and generate metaphors in the language context.

Comparative analysis of the semantics and grammar in the sacred context is of importance and many aspects of the difficulty dealing with the sacred words are better understood in a comparative context. Tournadre (2010) puts emphasis on the irregularities of describing grammar rules between cultures of different religions and analyzes the classical Tibetan cases and their trans-categoriality based on the treatises and grammars written specially for sacred books. White (1944) investigates the claim that certain words in American Indian languages are archaic and do not have any meaning. He reanalyzes these words and gets to the conclusion that these archaic words were the ceremonial vocabulary among Indian Pueblos, which have lost their meaning through the passage of history. Wardlaw (2008) investigates the meaning in terms of conceptualization, using a cognitive-semantic basis in the literary context. He uses the canonical analysis to semantically investigate the word God in Hebrew.

Languages behave differently based on their internal composition when it comes to the analysis of words in the sacred context. Hurst (1986) investigates the concept of "world language" composed of many sub-languages. He analyzes the alleged substratum of the words of Jesus in the Gospels and works through the principles affecting it. Singh (2018) investigates the mental processing involved in the spatial preposition formation, showing that mental processing differs for a variety of mental constructs. This shows that the sacred context can affect the way we think 
and we should consider the sacred context as separate from other ones. Borkowska and Kleparski (2007) investigate the pejoration as a semantic change which does affect not only the type of behavior we observe in people, but also it brings new meaning to the old unused word forms. Garnet (1990) investigates the New Testament translation issues, especially when they concern the concept of semantics and word choice in the framework of sacred texts. Salmond (1978) approaches the Maori world through the semantic analysis, concentrating on the pre-contact Maori sacred texts. Salmond brings different concepts, such as spatial prepositions, to the analysis, and proposes a cognitive-traditional concept in the Sacred Maori world. Furthermore, Singh R (2018) investigates the cognitive processing behind the inflectional phrase, showing how the semantics play an important role even in the most rigorous part of the generative grammar and he shows that concepts and semantics get formed in the mind in a hierarchical way.

Kim, de Marneffe, and Fosler-Loussier (2016) introduce the semantic intensity to improve vector-based semantic representations of words by adjusting word vectors. They bring the concept of semantic and meaning value to the core of the vector-based semantic representations. Engler and Gardiner (2017) look at four different perspectives on the 'sacred' in order to draw out their implicit, yet not surfaced prepositions about the meaning. They furthermore show that the views on sacred words rest on the implicit ground and this explains why certain theories about the sacred words can become contentious and problematic. Bentricia, Zidat, and Farhi (2017) investigate the semantics of the Quranic Arabic by extracting the semantics in an approach based on the Arabic conjunctive patterns.

Singh (2018) looks at the language change and proposes a model to account for the brutal language change. He brings forward the notion that conversion to another religion by the majority in a country does not account for the sudden language change and conceives the language change as a sign of the competition between languages, and the broader social context. This shows that morphology and the changes in words undergo situations where the context can influence the very language change. In the religious context, however, the situation is harder to explain. We have sacred words, still alive, but apparently no expert can easily trace their origin and even worse their very meaning is speculative.

\section{Sacred Words as Historical Relics}

It is true that many consider the words that are related to religions and cults as sacred and important. However, it is strange that many such words lose their semantic and symbolic meaning through the passage of time. The loss of semantics of cultural and religious words is a universal phenomenon and it happens almost regardless of the changes that happen in the religions of a country. If we consider India as a country with diverse religious configuration, we probably should have thought that such a phenomenon would have not happened in an old but yet alive religion such as Hinduism. But contrary to the expectations, even in India among Hindus, the true meaning and explanation of many of the Sanskrit words of the sacred Vedas are not available to the laymen. Furthermore, there is no consensus regarding the meaning of many sacred words among Hindu priests and scholars. Table 1 shows some sacred words from different languages that have lost their semantic meaning and are now considered as archaic and part of the rituals, or just sacred words without any clear link to their meaning and origin. Table 1 shows that the semantics and the origin of these sacred words are at best speculative and disputed, and yet these words are used every day by almost everyone in the society. Therefore, there is a dilemma: there are words that despite everyday use do not have a clear cut meaning.

Table 1. Sacred Words with Unknown or Disputed Origin and Semantics

\begin{tabular}{c|l|l|l}
\hline Sacred words & Language & $\underline{\text { Symbol }}$ & Origin and semantics \\
khoda & Persian & God & Not known \\
Gott & German & God & Not known \\
Indra & Sanskrit & God & Not known \\
Pushan & Sanskrit & God of marriage & Not known \\
Soma & Sanskrit & Vedic ritual drink & Speculative \\
Mungu & Swahili & God & Not known \\
Allah & Arabic & God & Speculative \\
Ange & French & Angel & Speculative \\
\hline
\end{tabular}




\subsection{Automaticity and Historical Semantic Loss Theory}

As discussed in the previous part, it is of interest to investigate why such sacred words have lost their semantics and origin among everyday people. We can find the reason in a simple phenomenon, which is called automaticity in the cognitive science. When we do something in a day to day life, we become skilled in doing it and cannot even notice how we do it. That is why describing how to ride a bicycle is hard, but we can show how to do it easily. When we go through the different sacred words, which are used in everyday life, we notice the same phenomenon. It is rather the collective automaticity, which is acquired by the majority of the society. It is as if though we as the society were not able to get out of the chain of automaticity. It is remarkable how the "chain of automaticity" works. Consider Fig.1 for a better understanding of the automaticity chain process. In fig.1, the plus sign is used to show that we have one entity in the sacred word and the negative sign shows its absence.

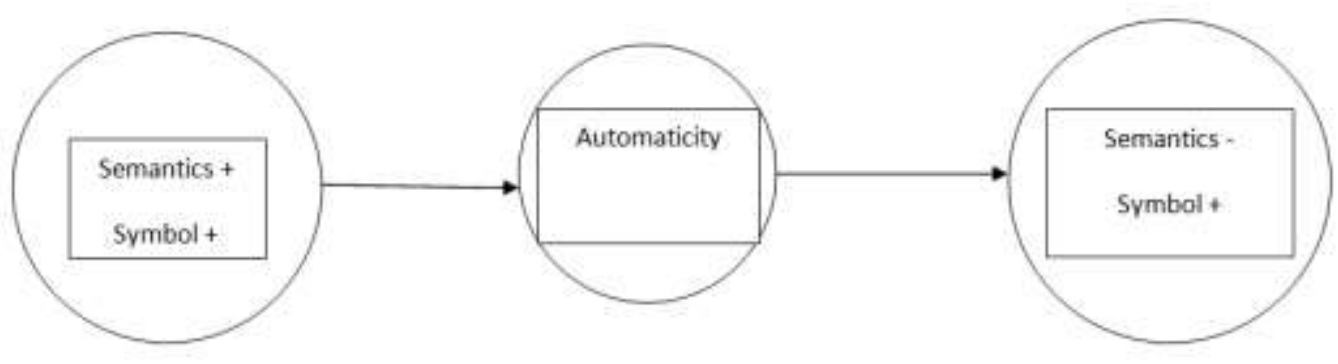

Figure 1. Historical trend and loss of semantics in the automaticity chain process

Automaticity affects every aspect of life, and it also shows a collective effect through the sacred words and their loss of semantics. An interesting question arises as whether we can have a model in which we recognize words which may lose their semantics through the automaticity process. Loss of semantics is an observable phenomenon which does not recognize frontiers, be it in Iran, India or the Americas. This simple fact shows that automaticity is a process which is hard to control. Thus we have a function which can be formulated as below in Formula 1.

1) $c=F(S Y, S E, A B)=S Y+S E+A B$

$$
A B=a(S Y)-b(S E)
$$

Where

$\mathrm{SE}=$ Semantic load

SY=Symbol load

which is constant $\mathrm{F}=$ Word Functional load

\section{$\mathrm{a}>1 \& \mathrm{~b}>1$}

As can be noted in the above formula, the abstraction influences the content of semantic and symbolic element of the word function. It is important to note the presence of coefficients $a$ and $b$ which denote the socio-contextual situation of the linguistic society. Words can stand anywhere between the absolute semantic word and absolute symbolic word, though no word can be at the extremes. Sacred words have high AB, abstraction, and therefore their function has higher symbolic load. Sacred words are close to the symbolic extreme, and the physical words are on the other end. The functional load is illustrated in figure 2. 


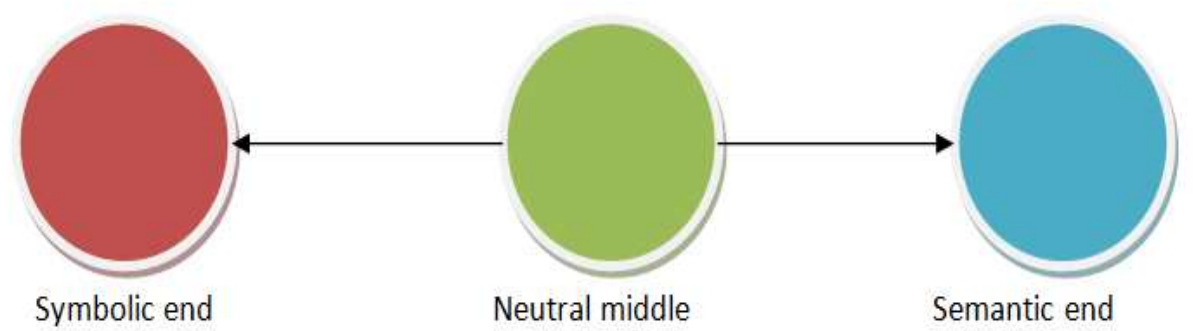

Figure 2. Functional load representation

\section{Word Automaticity Process Experiment}

\subsection{Participants and Experiment Method}

We tried to find how much the automaticity affects words by two simple experiments. We asked 10 volunteers to use 5 artificially constructed words for one. In order to monitor the experiment better, we chose a well-knit group of people. This way, we could monitor and observe how much and how often they were using these words in their casual meetings. It was important to have participants who we can monitor for one year in order to ensure for the reliability and validity of our experiments. The participants were of the same gender ratio, and they were between 28 and 44. The words were made from the Persian familiar words, so that semantics still could be relevant. Table 2 shows these words and the percentage of participants who could still remember their semantics after 1 year of usage. Below we show how we made these words.

Khobar: khoob(good)+bar(load)=goodness

Rashonoo: rah (road)+shenoo (listen and follow)=guidance

Rahadel: Raha (free)+ del ( heart) $=$ sacrifice

Cheshko: cheshm (eye)+ khoob (good)= beauty

Darmah $=$ darya $($ sea $)+$ mah $($ moon $)=$ Nature

Nejra: Nejat (save) + rah (road)= prophet or success

We further split the group in half and gave them the same word (Nejra) with a different meaning for the two groups. The result of the second experiment is given in Table 3, which is done concurrently with the first experiment.

Table 2. Meaning-Based Made Words and Semantic Loss

\begin{tabular}{lll}
\hline Constructed words & Meaning & $\underline{\%}$ \\
Khobar & goodness & 13 \\
Rashonoo & Guidance & 18 \\
Rahadel & Sacrifice & 40 \\
cheshkho & beauty & 25 \\
darmah & nature & 18 \\
\hline
\end{tabular}

Note. \% is the percentage of participants who remember the semantics of the given word after 1 year.

Table 3. Meaning-Based Made Words and Semantic Loss for Smaller Groups of 25

$\begin{array}{lll}\text { Constructed words } & \text { Meaning } & \underline{\%} \\ \text { Nejra } & \text { Prophet } & 26 \\ \text { Nejra } & \text { success } & 10\end{array}$

Note. $\%$ is the percentage of participants who remember the semantics of the given word after 1 year. The initial group is divided into two groups of 25 people with Nejra having a different meaning for each of them. 


\subsection{Analysis of the Semantic Loss Experiments}

We further divided these meanings-based artificial words into two broad groups, i.e. the abstract words and the concrete words. Table 4 shows the broad division of the words into abstract and concrete words, and the analysis of the number of participants who remembered the semantics of these words after three years of day to day usage.

Table 4. Abstract and Concrete Words Division

\begin{tabular}{llll}
\hline Abstract words & $\underline{\%}$ & $\underline{\text { Concrete words }}$ & $\underline{\%}$ \\
Khobar (goodness) & 12 & Darmah (nature) & 18 \\
Nejra (success) & 10 & Nejra (prophet) & \\
Rahadel (sacrifice) & 40 & \\
Cheshko (beauty) & 25 & \\
Rashonoo & 12 & \\
(guidance) &
\end{tabular}

Note. \% is the percentage of participants who remember the semantics of the given word after 1 year.

When we look at the abstract/concrete distinction, we see that overall the concrete words are much higher than their abstract counterparts, with the exception of Rahadel (sacrifice) and Cheshko (beauty). This means that abstract words are harder to conceive and therefore they have a stronger symbolic significance for their social users compared to the concrete words that show their efficacy when required. Though for our analysis to be more convincing, we need to explain why sacrifice and beauty, though rather abstract, still have pretty high percentage of participants who could remember their semantics after one year. For rahadel, it is its simple and compound system that makes it easier to remember semantically. Rahadel is a compound noun made of raha and del, so clearly compound abstract nouns are closer to concrete words cognitively. Furthermore, Cheshko is an abstract noun that have a strong relationship between its components, beautiful eye, consisting of cheshm(eye) and khoob(good), thus it is remembered better. So we can again consider cheshko as a compound noun since its component are readily discernable in Persian.

It is then significant to see how abstract words are more influenced by the automaticity. We know that sacred words are used by everyone in a rather abstract way. Even the word "God" is very abstract in usage, shapeless, and it is hard to define its concrete points in space. Thus, sacred words do act like abstract nouns in every sense and they lose their semantics because of this very abstract nature. This also implies that the mind begins the abstraction process combined with the automaticity. After doing the experiment, it got clear how the automaticity is complex and dependent on the abstract nature of words - when words are of abstract nature, they render themselves more readily to the process of automaticity. This is the clear reason why sacred words often pose problems of translation.

The real challenge for translators is to recognize the very fact that sacred words are devoid of their true origin and meaning, showing rather a symbolic function. Thus, the dichotomy of symbol/semantics in its own right deserves further investigation. From our experiments, it is clear that in sacred words context, this dichotomy is salient, contrasting with the rest of literature world where symbol is attached inherently with the semantics as a clarifier.

\section{Conclusion and Discussion}

Language behaves in a non-linear fashion and we see this non-linearity in the word formation and abstraction of the sacred words. Semantics is deeply related to the mental representation for concepts, but sometimes this linear one-to-one matching becomes distorted by the introduction of other phenomena, less susceptible to get attention. This situation is best observed in the context where notions lose their semantics and the core of meaning, leaving symbolic representation as the ideal form to be retained in the heart of the religious world. Automaticity then influences the way we think more than what was expected before this study. We showed how automaticity begins to play with the notions, highlighting symbolic function. This is consistent with the fact that religious concepts are hard to conceive and explain. One can talk about physical shapes and instruments easier than far-fetched notions such as God and angels. Thus, we can have a better, context related translation procedure, taking into consideration the automatic abstraction of the sacred words. Moreover, the loss of semantics shows that the mind also makes use of special mental instruments to remain efficient, especially when it comes to the memory load.

Abstraction is a way to have a top-down direction to the mental word processing, instead of the bottom-up one - the mind does not search for the meaning of the sacred words and it uses the symbolic presentation to process them. The whole abstraction process is designed in mind in a way that the processing load on the working memory decreases. This is an important finding since mental mechanisms are hard to control and to account for in previous models. 
Sacred words indeed show this irregular abstraction processing in mind, which makes them more readily understandable without a clear definition.

\section{References}

Bentricia, R., Zidat, S., \& Farhi, M. (2017). Extracting semantic relations from the Quranic Arabic based on Arabic conjunctive pattern. Journal of King Saud University - computer and information sciences (articles in press). https://doi.org/10.1016/j.jksuci.2017.09.004

Borkowska, P. \& Kleparski, G. A. (2007). it befall words to fall down: pejoration as a type of semantic change. zezyt 47 (2007): 33-50. www.ur.edu.pl . Uniwersytetu Rzeszowskiego, 2007. Web. 05 Apr. 2015. Retrieved from: https://www.ur.edu.pl/file/1294/sar_v4_03.pdf

Engler, S., \& Gardiner, W. (2017). Semantics and the Sacred. Religion, 47(4), 616-640. https://doi.org/10.1080/0048721x.2017.1362784

Garnet, P. (1990). The concept of sacred language: Help or hindrance in New Testament translation?. Traduction, Terminologie, redaction, 3, 71-79. https://doi.org/10.7202/037069ar

Hurst, L., D. (1986). The neglected role of semantics in the search for the Aramaic words of Jesus. JSNT, 28, 63-80. https://doi.org/10.5040/9781472550101.ch-012

Kim, J. K., de Marneffe, M. C., \& Fosler-Loussier, E. (2016). Adjusting Word Embeddings with Semantic Intensity Orders. in ACL 2016 workshop on representation learning for NLP (RepL4NLP), 2016, 62-69. https://doi.org/10.18653/v1/w16-1607

Salmond, A. (1978). Te Ao Tawhito: A semantic approach to the traditional Maori Cosmos. New Zealand Journal of The Polynesian Society, 87, 5-28. https://doi.org/10.26686/jnzs.v0i25.4110

Singh, R. (2018). Norwegian and Persian spatial prepositions: a novel comparative and syntactic approach to the spatial prepositions. KAAV international journal of English, literature, and linguistics, 5, 1-8. Retrieved from: http://www.kaavpublications.org/journals/journal-5/abstract/abstract-1788.pdf

Singh, R. (2018). Language change and directionality: A novel model for language change in Persian and Iranian languages from the sociolinguistic perspective. KAAV International journal of English, Literature, and linguistics, 5, 1-8. Retrieved from: www.kaavpublications.org/journals/journal-5/article/article-1876.pdf

Singh, R. (2018). Derivational Grammar Model and Basket Verb: A Novel Approach to the Inflectional Phrase in the Generative Grammar and Cognitive Processing. English Linguistics Research, 7(2). https://doi.org/10.5430/elr.v7n2p9

Tambiah, S. J. (1968). The magical power of words. Man, n.s. 3(2), 175-208. https://doi.org/10.2307/2798500

Tournadre, N. (2010). The Classical Tibetan cases and their transcategoriality. From sacred grammar to modern linguistics. Himalayan Linguistics, 9(2), 87-125. Retrieved from: https://escholarship.org/uc/item/94d0447c

Veal, T., \& Hao, Y. (2008). A fluid knowledge representation for understanding and generating creative metaphors. In Harold Somers (ed.), COLING 2008. Proceedings of the $22^{\text {nd }}$ International Conference on Computational Linguistics 945-952. Manchester: University of Manchester. https://doi.org/10.3115/1599081.1599200

Wardlaw, T., R. (2008). Conceptualizing words for God within the Pentateuch: a cognitive Semantic investigation in literary context. New York, London: T\&T Clark. Retrieved from: eprints.glos.ac.uk/3158/1/432407_Redacted.pdf

White, L. (1944). A ceremonial vocabulary among the pueblos. International Journal of American Linguistics, 10, 161-167. https://doi.org/10.1086/463838 